\title{
Overexpression of forkhead box M1 and urokinase-type plasminogen activator in gastric cancer is associated with cancer progression and poor prognosis
}

\author{
JIE MA ${ }^{1}$, GUANGWEI $\mathrm{QI}^{2}, \mathrm{JI}_{\mathrm{XU}}^{3}{ }^{3}$, HAIBING NI $^{4}$, WULIN XU ${ }^{5}$, \\ GUOQING RU ${ }^{1}$, ZHONGSHENG ZHAO ${ }^{1}$, WENJUAN XU ${ }^{1}$ and XUJUN HE ${ }^{6}$
}

\begin{abstract}
${ }^{1}$ Department of Pathology, Zhejiang Provincial People's Hospital, People's Hospital of Hangzhou Medical College;
${ }^{2}$ Department of Pathology, Hangzhou Children's Hospital; ${ }^{3}$ Department of Surgery, Zhejiang Provincial People's Hospital, People's Hospital of Hangzhou Medical College, Hangzhou, Zhejiang 310014; ${ }^{4}$ Department of Surgery, Tongde Hospital of Zhejiang, Hangzhou, Zhejiang 310012; ${ }^{5}$ Department of Respiratory Medicine;

${ }^{6}$ Key Laboratory of Gastroenterology of Zhejiang, Zhejiang Provincial People's Hospital, People's Hospital of Hangzhou Medical College, Hangzhou, Zhejiang 310014, P.R. China
\end{abstract}

Received July 10, 2016; Accepted July 3, 2017

DOI: $10.3892 / \mathrm{ol} .2017 .7136$

\begin{abstract}
Forkhead box M1 (FOXM1) and urokinase-type plasminogen activator (uPA) are overexpressed and associated with the pathogenesis of multiple types of human malignancy. The aims of the present study were to investigate FOXM1 and uPA expression levels in human gastric cancer using tissue microarray techniques; determining their association with clinicopathological characteristics as well as their prognostic value. Tissue microarray blocks, comprising 436 gastric cancer cases and 92 non-cancerous adjacent normal gastric tissues, were analyzed for FOXM1 and uPA protein expression levels using immunohistochemistry. The results were analyzed statistically in association with various clinicopathological characteristics and overall survival rates. FOXM1 and uPA were detected in 78.67 (343/436) and 83.26\% (363/436) of cancer samples, respectively. FOXM1 and uPA were not expressed in the 92 normal gastric tissue samples. In gastric cancer, FOXM1 and uPA levels were associated with tumor size, depth of invasion,
\end{abstract}

Correspondence to: Dr Xujun He, Key Laboratory of Gastroenterology of Zhejiang, Zhejiang Provincial People's Hospital, People's Hospital of Hangzhou Medical College, 158 Shangtang Road, Hangzhou, Zhejiang 310014, P.R. China

E-mail: hxj0105099@163.com

Dr Wenjuan Xu, Department of Pathology, Zhejiang Provincial People's Hospital, People's Hospital of Hangzhou Medical College, 158 Shangtang Road, Hangzhou, Zhejiang 310014, P.R. China E-mail: 694386617@qq.com

Key words: gastric cancer, forkhead box protein M1, urokinase-type plasminogen activator, tissue microarray, cancer progression, prognosis tumor-node-metastasis (TNM) stage, lymph node metastasis, vessel invasion and distant metastases. The overall survival rate was significantly decreased in patients expressing FOXM1 and uPA compared with FOXM1- and UPA-negative patients. Cox's multivariate analysis revealed that age, depth of invasion and expression levels of FOXM1 and uPA are independent predictors of survival in patients with gastric cancer. These results indicated that increased FOXM1 and uPA expression levels are associated with the invasive and metastatic processes in human gastric cancer, and inversely associated with patient prognosis. Therefore, FOXM1 and uPA may serve as novel prognostic markers independent of, but supplementing, the TNM staging system.

\section{Introduction}

Gastric cancer, one of the most common malignancies of the digestive system, is the second leading cause of cancer-associated mortality worldwide, with an increased frequency in East Asia, particularly in China (1). Despite the decreased incidence and mortality of gastric cancer in China over the past decades, this malignancy remains a prominent burden to local health programs (2). Currently, surgical resection is the only curative treatment available for localized gastric cancer; however, nearly half of patients diagnosed possess advanced to late-stage cancer, with lymph node or distant metastasis, and are not eligible for surgery $(3,4)$. Among the patients presenting with early-stage disease, even following a potentially curative resection, $60 \%$ eventually develop distant metastasis or local recurrence (5). The prognosis of patients with advanced or recurrent gastric cancer remains poor, with 5-year survival rates ranging between 5 and $17 \%$ in the Western world, and have not changed significantly in the last 30 years (6-8). Thus, identification of novel biomarkers for early diagnosis or prognosis is key to improving the prognosis of patients with gastric cancer. 
Forkhead box M1 (FOXM1), characterized by a 100-amino-acid winged-helix DNA-binding domain, is a member of the forkhead transcription factor family. It serves an important role in cell cycle regulation by promoting the transition from $\mathrm{G} 1$ to $\mathrm{S}$ phase and progression to mitosis through cell division cycle 25 homolog $\mathrm{B}$, cyclin-dependent kinase 1 and cyclin-dependent kinase inhibitor 1B (1,9-11). Recent studies have demonstrated that FOXM1 is overexpressed and serves an essential role in the development and progression of distinct types of human cancer, including hepatocellular carcinoma, non-small cell lung cancer and breast cancer (12-14). In a rhabdomyosarcoma cell line, FOXM1 silencing results in decreased cell growth and survival (15).

Urokinase-type plasminogen activator (uPA), a member of the uPA system, is a trypsin-like serine protease. The uPA ligand binds to its receptor, a three-domain glycolipid-anchored cell-surface protein, and mediates proteolysis by plasminogen or growth factor activation (16-19). Previous studies have demonstrated that increased expression of the uPA system in tumor tissues is associated with pathological characteristics and prognosis in numerous types of tumor due to its roles in cell adhesion, migration and invasion, as well as metastasis $(17,20,21)$. Plebani et al (22) investigated uPA levels in gastric cancer and normal samples from 20 patients with gastric cancer using ELISA and identified significantly increased uPA expression levels in cancer samples, with decreased uPA receptor levels identified to be associated with a prolonged survival time.

Although FOXM1 and uPA are considered to mediate tumor cell differentiation, invasion and metastasis in human cancer, the association of their expression levels with clinicopathological characteristics in patients with gastric cancer remains unknown. Similarly, the importance of FOXM1 expression and its effect on uPA in gastric cancer biology as well as patient prognosis are not well-understood. Therefore, the aim of the present study was to investigate FOXM1 and uPA expression levels in gastric tissue specimens and evaluate their association with the clinicopathological features of gastric cancer as well as exploring their individual or combined effects on the clinical outcome. The results of the present study provide a basis for understanding the clinical significance of FOXM1 and uPA in the prognosis of patients with gastric cancer.

\section{Materials and methods}

Patients and tissue samples. A total of 436 patients (310 males and 126 females; median age, 59.50 years; range, 17-99 years) with gastric cancer, who underwent gastrectomy in Zhejiang Provincial People's Hospital (Hangzhou, China) between March 1998 and December 2004, were consecutively enrolled in the present study. Written informed consent was obtained from each patient and the present study was approved by the Ethics Committee of Zhejiang Provincial People's Hospital. All patients met the following eligibility criteria: i) Tumor histologically confirmed to be gastric cancer; ii) no previous endoscopic mucosal resection, palliative resection, preoperative chemotherapy or radiotherapy; and iii) 5 years of follow-up data available, with follow-up ending in December 2008. Clinicopathological data were obtained from operative and pathological reports, including tumor location, size, Lauren type (23), histological classification, degree of differentiation, depth of tumor invasion, tumor-node-metastasis (TNM) stage, lymph node status, vessel invasion and distant metastasis status. All cases were classified according to the World Health Organization's pathological classification (2010) of gastric tumors (23). Survival time was defined as between the date of surgery and the follow-up deadline or date of mortality. In addition, 92 para-cancer tissues, $>5 \mathrm{~cm}$ away from the tumor lesion boundary, were randomly selected as controls. All tissue samples were fixed in $10 \%$ neutral formalin buffer $(\mathrm{pH} 7.4)$ at room temperature for $10 \mathrm{~h}$, paraffin-embedded (FFPE), and stored until used.

Tissue microarray (TMA). TMAs were constructed using FFPE blocks from 436 tumor tissue samples and 92 normal mucosa specimens; all experiments were performed on an automated TMA instrument. Briefly, 4- $\mu$ m-thick sections were obtained from each tissue block and stained with hematoxylin and eosin for $10 \mathrm{~min}$ at room temperature to identify appropriate tumor areas. Core tumor tissue biopsies (diameter, $2 \mathrm{~mm}$ ) were obtained from individual FFPE blocks (donor blocks) and arranged in recipient paraffin blocks (tissue array blocks) using a trephine. Each TMA block contained $>6$ adjacent non-cancerous mucosal specimens serving as internal controls.

Immunohistochemical staining and evaluation. Immunohistochemical staining was performed to analyze the expression of FOXM1 and UPA in 4- $\mu$ m-thick TMA sections including 436 gastric cancer and 92 adjacent non-cancerous tissue samples. Briefly, TMA sections were deparaffinized twice for $15 \mathrm{~min}$ in xylene, rehydrated in graded ethanol and incubated in $3 \% \mathrm{H}_{2} \mathrm{O}_{2}$ at room temperature for 15 min to block endogenous peroxidase activity. The sections were microwaved in $0.01 \mathrm{M}$ of citrate buffer (1.92 $\mathrm{g}$ anhydrous citric acid dissolved in 1 liter distilled water and adjusted to $\mathrm{pH} 7.4$ with $1 \mathrm{~N} \mathrm{NaOH}$ ), boiled for $15 \mathrm{~min}$ for antigen retrieval, and incubated with $10 \%$ normal goat serum (cat. no. 50062Z; Thermo Fisher Scientific Inc., Waltham, MA, USA) at room temperature for $10 \mathrm{~min}$ to block non-specific binding. Subsequently, the sections were incubated with rabbit monoclonal anti-FOXM1 (1:500 dilution in PBS; ab207298; Abcam, Cambridge, MA, USA) and anti-uPA (1:250 dilution in PBS; ab169754; Abcam) antibodies overnight at $4^{\circ} \mathrm{C}$, with normal goat serum used as a negative control. Following incubation with horseradish peroxidase-conjugated anti-rabbit secondary antibodies (cat. no. ZDR-5306; OriGene Technologies, Inc., Rockville, MD, USA) for $20 \mathrm{~min}$ at room temperature, 3'-diaminobenzidine tetrahydrochloride was used to visualize the signals. Counterstaining was performed with hematoxylin, staining for $10 \mathrm{~min}$ at room temperature. Immunohistochemical evaluation was independently performed by two pathologists blinded to the clinical data. Average staining intensity in each case was classified into one of the following four categories: 0, Negative; 1, weak staining; 2 , moderate staining; 3 , intense staining. Meanwhile, the proportion of cells with positive-staining were classified as follows: $0,<5 \% ; 1,5-25 \% ; 2,26-50 \% ; 3,51-75 \%$; $4,76-100 \%$. The overall staining index was calculated as the sum of the staining intensity score and proportion of positively stained cells. The final composite scores were divided into 
four grades: 0-1 (-), 2-3 (+), 4-6 (++) and $6(+++)$. Patients with a score of '-' were classified as the negative expression group, whereas those with,+++ and +++ comprised the low, moderate and high expression groups, respectively. Finally, low, moderate and high expression groups were all considered to be FOXM1- and UPA-positive.

Statistical analysis. All statistical analyses were performed using SPSS (version 13.0; SPSS Inc., Chicago, IL, USA) and GraphPad Prism (version 5.01; GraphPad Software Inc., La Jolla, CA, USA). Categorical data were assessed using $\chi^{2}$ or Fisher's exact test. Survival curves were evaluated using the Kaplan-Meier estimator method, with the log-rank test used to assess group differences. Multivariate analysis using the Cox's proportional hazards regression model was performed to assess FOXM1 and uPA protein levels for their prognostic value. The associations of FOXM1 and uPA expression with clinicopathological characteristics were estimated using the Pearson's correlation method. Results are presented as the mean \pm standard deviaiton. $\mathrm{P}<0.05$ was considered to indicate a statistically significant difference.

\section{Results}

Clinicopathological characteristics. A total of 436 patients with gastric cancer (310 males and 126 females) were included in the present study. On the basis of the World Health Organization Classification Criteria (2010) for gastric cancer (23), the diagnoses included 28 mucinous adenocarcinomas, 16 papillary adenocarcinomas, 65 signet ring cell carcinomas and 327 tubular adenocarcinomas. A total of 3 cases were undifferentiated, 309 were poorly differentiated, 109 were moderately differentiated and 15 cases were well-differentiated. According to the TNM staging criteria, 90, 104, 173 and 69 cases were TNM stages I, II, III and IV, respectively; 270 cases exhibited lymph node metastasis, whereas 166 cases did not $(\mathrm{P}<0.05$; Table I).

Clinical pathology of FOXM1 and UPA expression in gastric cancer. FOXM1 and UPA were identified in cancer cells; in the 436 gastric cancer specimens, FOXM1 and uPA detection rates were $78.67(343 / 436)$ and $83.26 \%$ (363/436), respectively; however, only a limited number of stained tissues were observed among the 92 para-cancer tissues (Table II; Figs. 1 and 2). These results indicated that FOXM1 and uPA expression levels were significantly increased in gastric cancer compared with para-cancer tissues $(\mathrm{P}<0.05)$.

FOXM1 and uPA levels were associated with invasion depth, TNM stage, lymph node status, vessel invasion and distant metastasis $(\mathrm{P}<0.05$; Table I). In addition, the FOXM1 expression level was associated with differentiation and Lauren classification, and uPA expression was associated with tumor size $(\mathrm{P}<0.05$; Table I). However, no significant association between other clinicopathological characteristics and FOXM1 and UPA expression levels was identified.

The FOXM1 detection rate was increased in patients with lymph node metastasis $(88.5 \%, 239 / 270)$ compared with those without lymph node metastasis $(62.7 \%, 104 / 166$; $\mathrm{P}<0.05)$. The FOXM1 detection rate was also increased in patients with distant metastasis $(90.2 \%, 55 / 61)$ compared with those without
(76.8\%, 288/375; $\mathrm{P}<0.05)$. Patients with stage III and IV gastric cancer exhibited increased FOXM1 detection rates (86.1 and $91.3 \%$, respectively) compared with those with stage I and II gastric cancer (71.1 and 64.4\%, respectively; $\mathrm{P}<0.05$ ). Similarly, T3 and T4 stage patients exhibited increased FOXM1 detection rates ( 86.9 and $80.8 \%$, respectively) compared with $\mathrm{T} 1$ and $\mathrm{T} 2$ stage cases (66.7 and $66.1 \%$, respectively). Furthermore, the FOXM1 detection rate was $86.9 \%(220 / 253)$ in gastric carcinoma specimens with vessel invasion, which was increased compared with that obtained for specimens without vessel invasion $(67.2 \%, 123 / 183 ; \mathrm{P}<0.05)$. FOXM1 detection rates were increased in patients with intestinal and mixed types (81.7 and $95.1 \%$, respectively) compared with diffuse-type cases $(68.8 \% ; \mathrm{P}<0.05)$.

The uPA detection rate was increased in gastric carcinoma specimens with tumor size $\geq 5 \mathrm{~cm}$ at $67.6 \%(121 / 179)$, compared with tumor size $<5 \mathrm{~cm}(22.6 \%, 58 / 257 ; \mathrm{P}<0.05)$. The uPA detection rate was increased in patients with lymph node metastasis $(74.4 \%, 201 / 270 ; \mathrm{P}<0.05)$ compared with those without $(23.5 \%, 39 / 166 ; \mathrm{P}<0.01)$. Meanwhile, uPA detection rates were increased in specimens with vascular invasion and distant metastasis at $71.9 \%(182 / 253)$ and $91.8 \%(56 / 61)$, respectively, compared with cases without vascular invasion $(31.6 \%, 58 / 183 ; \mathrm{P}<0.05)$ or distant metastasis $(49.1 \%, 184 / 375$; $\mathrm{P}<0.05)$. In addition, uPA was detected in $18.8 \%$ (17/90) and $32.7 \%$ (34/104) of TNM stage I and II samples, respectively, which was decreased compared with TNM stage III and IV specimens ( $72.8 \%$ or $126 / 173$ and $91.3 \%$ or $63 / 69$, respectively; $\mathrm{P}<0.01)$. Notably, uPA expression was associated with depth of invasion in gastric cancer; from $\mathrm{T} 1$ to $\mathrm{T} 4$ stages, positive rates gradually increased (T1, 22.8\%; T2, 42.2\%; T3, 64.3\%; T4, 92.3\%; $\mathrm{P}<0.01$ ). No significant association between FOXM1 and $\mathrm{uPA}$ expression and the remaining clinicopathological parameters was identified. Cox's multivariate analysis revealed that age, TNM stage, distant metastasis and FOXM1 and UPA levels were independent prognostic factors (Table III). Thus, FOXM1 and uPA may be independent predictors of prognosis in patients with gastric cancer.

Increased FOXM1 and UPA expression levels are associated with poor prognosis. In the patient cohort of the present study $(\mathrm{n}=436)$, the mean survival time in FOXM1-positive patients (38.37 \pm 0.878 months) was significantly decreased compared with FOXM1-negative patients $(54.17 \pm 1.49$ months, $\mathrm{P}<0.01)$; the 5-year survival rate was also significantly decreased in FOXM1-positive patients $(16.9 \%, 58 / 343)$ compared with FOXM1-negative cases $(80.6 \%, 75 / 93$; P<0.01; Fig. 3A). FOXM1-positive patients exhibited decreased survival rates in the TNM grade I and II, and TNM grade III and IV groups, respectively, compared with patients not expressing these proteins $(\mathrm{P}<0.01$; Fig. 3B and C). Similarly, mean survival time in uPA-positive patients was significantly decreased compared with uPA-negative cases $(34.72 \pm 1.14$ vs. $50.37 \pm 0.83$ months; $\mathrm{P}<0.01)$; the 5-year survival rate was significantly decreased in uPA-positive patients $(19.6 \%, 47 / 240)$ compared with uPA-negative cases $(43.9 \%, 86 / 196 ; \mathrm{P}<0.01$; Fig. 3D). In the TNM grade I and II, and TNM grade III and IV groups, uPA-positive patients also exhibited decreased survival rates compared with patients not expressing these proteins $(\mathrm{P}<0.01$; Fig. 3E and F). 
Table I. Association of FOXM1 and uPA expression with various clinicopathological parameters in 436 patients with gastric cancer.

\begin{tabular}{|c|c|c|c|c|c|c|c|}
\hline \multirow[b]{2}{*}{ Variable } & \multirow[b]{2}{*}{ Patients (n, 436) } & \multicolumn{2}{|c|}{ FOXM1 } & \multirow[b]{2}{*}{ P-value } & \multicolumn{2}{|c|}{ uPA } & \multirow[b]{2}{*}{ P-value } \\
\hline & & Negative & Positive & & Negative & Positive & \\
\hline Age, years & & & & 0.210 & & & 0.041 \\
\hline$\leq 50$ & 344 & 69 & 275 & & 146 & 198 & \\
\hline$>50$ & 92 & 24 & 68 & & 50 & 42 & \\
\hline $\operatorname{Sex}$ & & & & 0.420 & & & 0.727 \\
\hline Male & 310 & 63 & 247 & & 141 & 169 & \\
\hline Female & 126 & 30 & 96 & & 55 & 71 & \\
\hline Location & & & & 0.187 & & & 0.471 \\
\hline Cardia & 59 & 10 & 49 & & 23 & 36 & \\
\hline Body & 171 & 44 & 127 & & 75 & 96 & \\
\hline Antrum & 206 & 39 & 167 & & 98 & 108 & \\
\hline Tumor size, $\mathrm{cm}$ & & & & 0.142 & & & $<0.001$ \\
\hline$<5$ & 257 & 61 & 196 & & 138 & 58 & \\
\hline$\geq 5$ & 179 & 32 & 147 & & 119 & 121 & \\
\hline Lauren classification & & & & $<0.001$ & & & 0.073 \\
\hline Intestinal & 251 & 46 & 205 & & 121 & 130 & \\
\hline Diffuse & 144 & 45 & 99 & & 63 & 81 & \\
\hline Mixed & 41 & 2 & 39 & & 12 & 29 & \\
\hline Histology classification & & & & 0.727 & & & 0.471 \\
\hline Papillary & 16 & 3 & 13 & & 6 & 10 & \\
\hline Tubular & 327 & 70 & 257 & & 150 & 177 & \\
\hline Mucinous & 28 & 4 & 24 & & 9 & 19 & \\
\hline Signet ring cell & 65 & 16 & 49 & & 31 & 34 & \\
\hline Differentiation & & & & 0.004 & & & 0.101 \\
\hline Weak & 15 & 8 & 7 & & 11 & 4 & \\
\hline Moderate & 109 & 22 & 87 & & 51 & 58 & \\
\hline Strong & 309 & 61 & 248 & & 132 & 177 & \\
\hline Undifferentiated & 3 & 2 & 1 & & 2 & 1 & \\
\hline Invasion depth & & & & $<0.001$ & & & $<0.001$ \\
\hline $\mathrm{T} 1$ & 57 & 19 & 38 & & 44 & 13 & \\
\hline $\mathrm{T} 2$ & 109 & 37 & 72 & & 63 & 46 & \\
\hline $\mathrm{T} 3$ & 244 & 32 & 212 & & 87 & 157 & \\
\hline $\mathrm{T} 4$ & 26 & 5 & 21 & & 2 & 24 & \\
\hline TNM stages & & & & $<0.001$ & & & $<0.001$ \\
\hline I & 90 & 26 & 64 & & 73 & 17 & \\
\hline II & 104 & 37 & 67 & & 70 & 34 & \\
\hline III & 173 & 24 & 149 & & 47 & 126 & \\
\hline IV & 69 & 6 & 63 & & 6 & 63 & \\
\hline Lymph node metastasis & & & & $<0.001$ & & & $<0.001$ \\
\hline No & 166 & 62 & 104 & & 127 & 39 & \\
\hline Yes & 270 & 31 & 239 & & 69 & 201 & \\
\hline Vessel invasion & & & & $<0.001$ & & & $<0.001$ \\
\hline No & 183 & 60 & 123 & & 125 & 58 & \\
\hline Yes & 253 & 33 & 220 & & 71 & 182 & \\
\hline Distant metastasis & & & & $<0.001$ & & & $<0.001$ \\
\hline No & 375 & 87 & 288 & & 191 & 184 & \\
\hline Yes & 61 & 6 & 55 & & 5 & 56 & \\
\hline
\end{tabular}

FOXM1, forkhead box protein M1; uPA, urokinase-type plasminogen activator; TNM, tumor-node-metastasis. 
Table II. FOXM1 and uPA expression levels in gastric cancer and paraneoplastic tissue specimens.

\begin{tabular}{|c|c|c|c|c|c|c|c|c|}
\hline \multirow[b]{2}{*}{ Variable } & \multicolumn{4}{|c|}{ FOXM1 } & \multicolumn{4}{|c|}{ uPA } \\
\hline & $\begin{array}{l}\text { Gastric } \\
\text { cancer } \\
\text { tissues }\end{array}$ & $\begin{array}{l}\text { Para- } \\
\text { cancer } \\
\text { tissues }\end{array}$ & $\chi^{2}$ & P-value & $\begin{array}{l}\text { Gastric } \\
\text { cancer } \\
\text { tissues }\end{array}$ & $\begin{array}{l}\text { Para- } \\
\text { cancer } \\
\text { tissues }\end{array}$ & $\chi^{2}$ & P-value \\
\hline Negative & 93 & 75 & 127.026 & $<0.001$ & 73 & 78 & 172.806 & $<0.001$ \\
\hline \multicolumn{9}{|l|}{ Positive } \\
\hline Weak & 174 & 10 & & & 193 & 10 & & \\
\hline Moderate & 112 & 5 & & & 89 & 3 & & \\
\hline Strong & 57 & 2 & & & 81 & 1 & & \\
\hline Total & 436 & 92 & & & 436 & 92 & & \\
\hline
\end{tabular}

FOXM1, forkhead box protein M1; uPA, urokinase-type plasminogen activator.

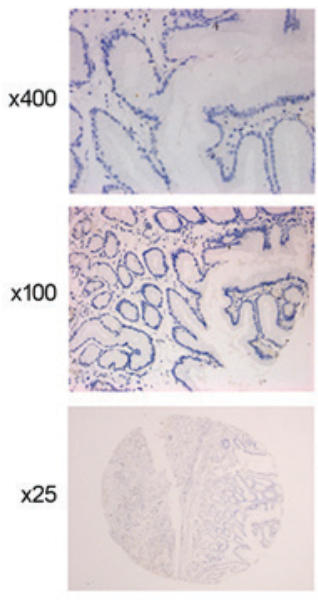

Normal (-)

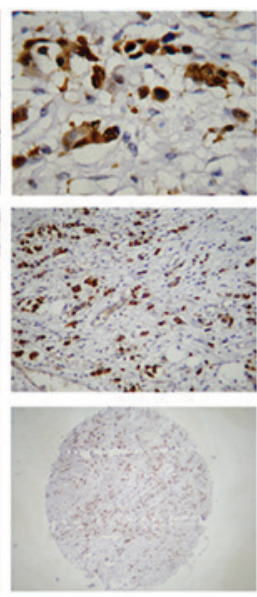

Poorly differentiated

Adenocarcinoma (+++)

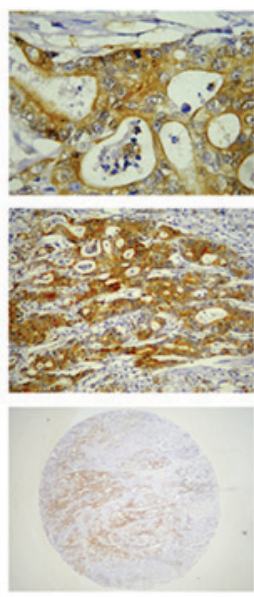

Moderately differentiated

Adenocarcinoma $(+++)$

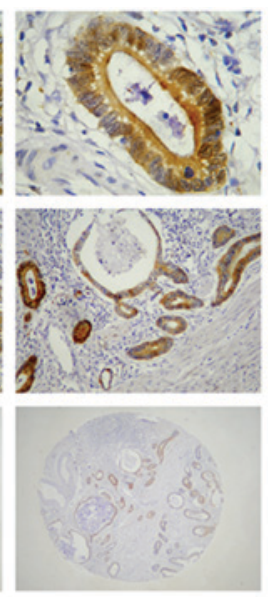

Well differentiated

Adenocarcinoma (+++)

Figure 1. Representative images of forkhead box protein M1 immunohistochemical staining in gastric cancer and normal mucosal tissues (magnification, $\mathrm{x} 25$, $\mathrm{x} 100$ and $\mathrm{x} 400$ ). No expression is represented by-, and high expression is represented by +++ .

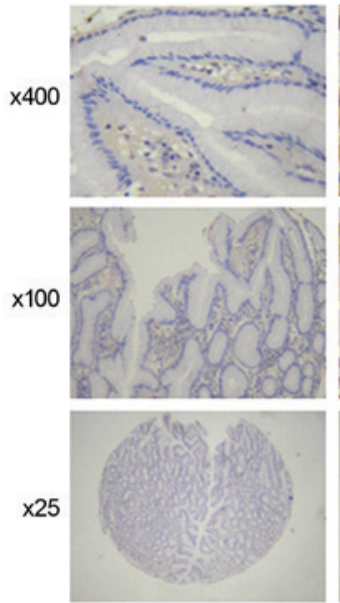

$\operatorname{Normal}(-)$
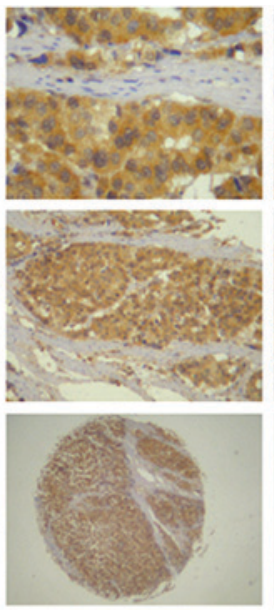

Poorly differentiated

Adenocarcinoma $(+++)$
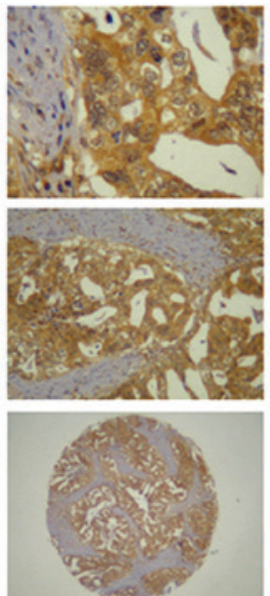

Moderately differentiated

Adenocarcinoma $(+++)$
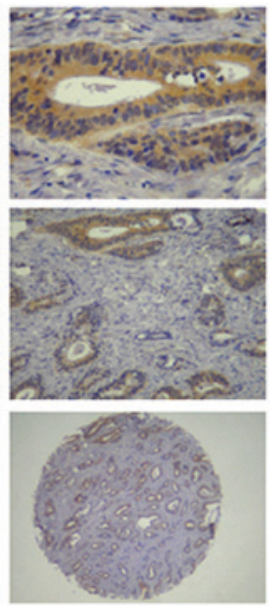

Well differentiated

Adenocarcinoma $(+++)$

Figure 2. Representative images of urokinase-type plasminogen activator immunohistochemical staining in gastric cancer and normal mucosal tissues (magnification, x25, x100 and x400). No expression is represented by-, and high expression is represented by +++ . 
Table III. Multivariate Cox's proportional hazard analysis of overall survival.

$95 \%$ CI for HR

\begin{tabular}{lccrcrrr}
\cline { 5 - 7 } Covariates & B-value & SE & Wald & P-value & HR & Lower & Upper \\
\hline Age & 0.012 & 0.005 & 4.872 & 0.027 & 1.012 & 1.001 & 1.023 \\
TNM stage & 0.565 & 0.143 & 15.711 & 0.000 & 1.760 & 1.331 & 2.327 \\
Distant metastasis & 0.463 & 0.206 & 5.064 & 0.024 & 1.589 & 1.062 & 2.379 \\
FOXM1 expression & 3.220 & 0.837 & 14.789 & 0.000 & 25.023 & 4.89 & 129.125 \\
uPA expression & 0.969 & 0.455 & 4.533 & 0.033 & 2.635 & 1.080 & 6.428 \\
Differentiation & 0.270 & 0.136 & 3.919 & 0.048 & 1.309 & 1.003 & 1.710 \\
\hline
\end{tabular}

FOXM1, forkhead box protein M1; uPA, urokinase-type plasminogen activator; CI, confidence interval; SE, standard error; HR, hazard ratio.

A

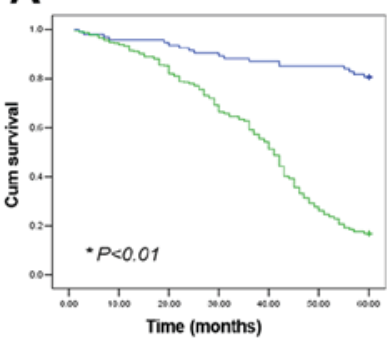

D

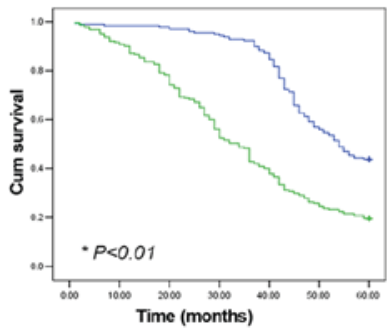

B

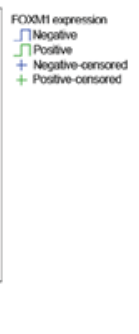

E

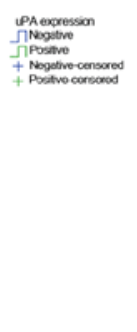

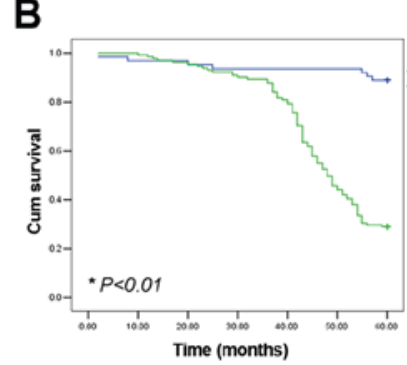

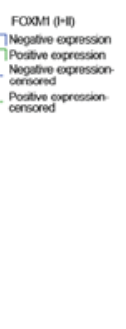

C

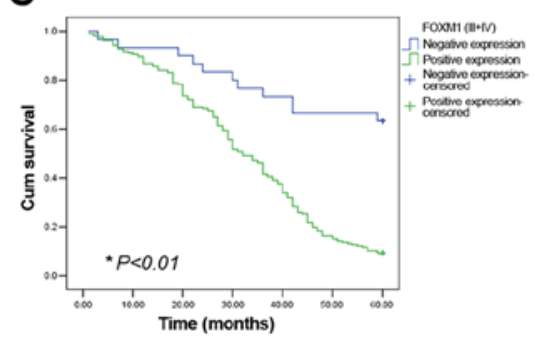

$\mathbf{F}$
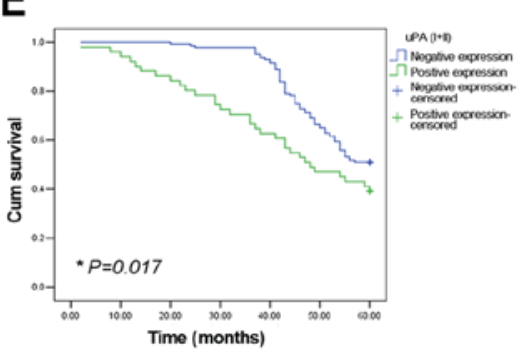

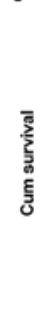

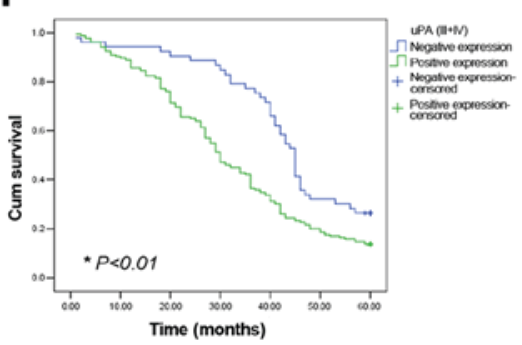

Figure 3. Kaplan-Meier estimator survival curves of patients with gastric cancer, positive or negative for FOXM1 and uPA. (A) FOXM1-positive or -negative patients. The 5-year survival rate in FOXM1-positive patients was significantly decreased compared with FOXM1-negative patients. (B) FOXM1-positive or -negative patients in TMN stages I and II. The 5-year survival rate in FOXM1-positive patients was significantly decreased compared with FOXM1-negative patients. (C) FOXM1-positive or -negative patients in TNM stages III and IV. The 5-year survival rate in FOXM1-positive patients was significantly decreased compared with FOXM1-negative patients. (D) uPA-positive or -negative patients. The 5-year survival rate in uPA-positive patients was significantly decreased compared with uPA-negative patients. (E) UPA-positive or -negative patients in TNM stages I and II. The 5-year survival rate of uPA-positive patients was significantly decreased compared with uPA-negative patients. (F) uPA-positive or -negative patients in TNM stages III and IV. The 5-year survival rate of uPA-positive patients was significantly decreased compared with uPA-negative patients (all $\mathrm{P}<0.05$ ). Roman numerals represent TNM stage. TNM, tumor-node-metastasis; FOXM1, forkhead box protein M1; uPA, urokinase-type plasminogen activator; cum, cumulative.

Association of FOXM1, uPA and co-expression with patient prognosis. There were 63 gastric cancer cases not expressing either FOXM1 or UPA, and 210 patients with gastric cancer expressing both proteins simultaneously. A total of 126 gastric cancer cases were positive for only FOXM1; furthermore, 37 gastric cancer cases only exhibited uPA expression. Mean 5-year survival in patients with uPA and FOXM1 double-negative cancer $(84.1 \%, 53 / 63)$ was markedly increased compared with cases expressing only uPA $(59.5 \%, 22 / 37)$ or FOXM1 $(26.2 \%$, $33 / 126)$, and markedly increased compared with patients with uPA and FOXM1 double-positive cancer (11.9\%, 25/210). The mean survival of uPA and FOXM1 double-positive patients was significantly decreased compared with uPA and FOXM1 double-negative patients (32.62 \pm 1.13 vs. $56.44 \pm 1.36$ months; $\mathrm{P}<0.01$; Fig. 4A). In the TNM grade I and II, and TNM grade III and IV groups, uPA and FOXM1 double-positive patients also exhibited a poorer prognosis compared with double-negative or single-positive patients $(\mathrm{P}<0.05$; Fig. $4 \mathrm{~B}$ and $\mathrm{C})$.

\section{Discussion}

The results of the present study indicated that FOXM1 and uPA expression levels were significantly increased in gastric cancer compared with para-cancerous tissues. Notably, increased FOXM1 and uPA protein levels were associated with clinicopathological factors such as tumor size, depth of invasion, 
A

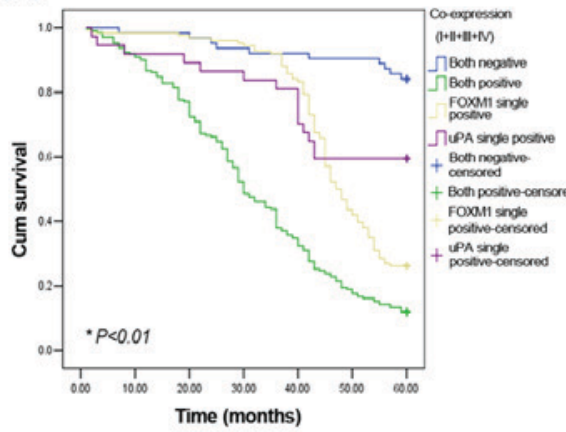

B

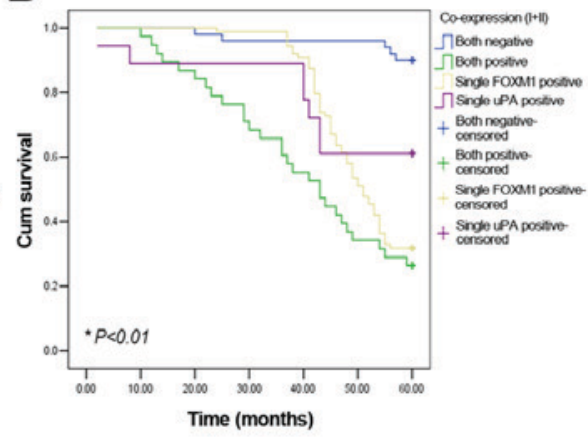

C

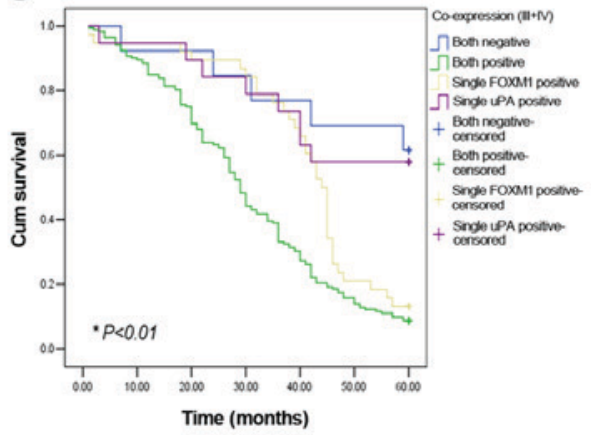

Figure 4. Kaplan-Meier estimator survival curves of patients with gastric cancer positive for FOXM1 and uPA. (A) The 5-year survival rate of FOXM1 and uPA double-positive patients was significantly decreased compared with patients not expressing these proteins in: (A) All cases; (B) TNM stages I and II; and (C) TNM stages III and IV. (all P<0.05). Roman numerals represent TNM stage. TNM, tumor-node-metastasis; FOXM1, forkhead box protein M1; uPA, urokinase-type plasminogen activator; cum, cumulative.

TNM stage, lymph node, vessel invasion and distant metastasis. In addition, patients with gastric cancer with increased FOXM1 and uPA levels exhibited poorer prognosis compared with decreased FOXM1 and uPA expression. Multivariate Cox's proportional hazards analysis revealed that age, invasion depth, FOXM1 level and uPA content were all independent indicators for overall survival time in gastric cancer. These results indicated that FOXM1 and UPA protein overexpression in gastric cancer is associated with malignant behavior and poor overall survival.

The FOXM1 gene ( $\sim 25 \mathrm{~kb})$, located at the 12p13-3 chromosome region and composed of 10 exons, exhibits the common characteristics of the family of FOX transcription factors: A conserved DNA sequence with a winged-helix domain (24). Important functions of FOXM1 include regulation of the cell cycle, promotion of cell proliferation, and inhibition of cell aging and apoptosis (9). Pathological overexpression of FOXM1 may induce the malignant proliferation of tumor cells (25). In contrast with the results of the present study, Okada et al (26) and $\mathrm{Li}$ et al (27) identified no significant association between FOXM1 overexpression and clinicopathological factors including pathological $\mathrm{T}$ factor, nodal involvement and histological differentiation. A possible reason for this discrepancy is that these studies comprised small sample sizes, and may not have allowed adequate exploration of the association between FOXM1 expression and clinicopathological factors. In the present study, FOXM1 expression was identified to be significantly associated with the prognosis of patients with gastric cancer; patients with increased FOXM1 expression exhibited a decreased survival time compared with those not expressing FOXM1. Hui et al (28) identified that patients with esophageal squamous cell carcinoma with nuclear FOXM1 expression are younger compared with those without nuclear expression. Therefore, there is a requirement for more detailed age-specific analyses to clarify the association between age and FOXM1 expression in human cancer.

FOXM1 upregulation has been detected in a broad range of human cancer cell lines and cancer types, indicating that FOXM1 is associated with aggressive behavior of tumor cells in vitro $(24,29,30)$. The results of the present study provided further support to this hypothesis, revealing FOXM1 to be an independent prognostic factor in gastric cancer. Previous studies indicated that other factors may be more significant than FOXM1 in predicting prognosis in early disease stage, including tumor location and lymph node metastasis; whereas in advanced stages, angiogenesis, which is promoted by FOXM1, may influence tumor growth rates more significantly $(30,31)$. The results of the present study suggested that FOXM1 expression in gastric cancer serves an unfavorable prognostic role in stage I to IV patients, supporting the hypothesis formulated by the aforementioned studies.

Cancer cell invasion is accomplished by the concerted action of a number of extracellular proteolytic enzyme systems, including the uPA system, which is one of the most important. Although referred to as a kinase, uPA possesses no kinase activity and in turn, by limited proteolysis, may be converted into active uPA (32). Previous studies using a variety of models have demonstrated that UPA is causally involved in promoting cancer invasion and metastasis. For example, Bekes et al (33) revealed that uPA participates in an early phase of prostate cancer dissemination (i.e. the initial escape of tumor cells from the primary site). In addition, Tang and Han (34) reported that the uPA system serves an important role in breast cancer growth, invasion and metastasis, possibly through the Ras/extracellular-signal-regulated kinase or p38 mitogen-activated protein kinase signaling pathway. Similarly, Heiss et al (35) demonstrated that uPA receptor expression on the tumor cell surface may be considered an independent biomarker for predicting bone marrow micro-metastasis in gastric cancer. As demonstrated by the results of the present study, uPA expression was significantly increased in gastric cancer compared with para-cancer tissues, and was associated with depth of invasion, TNM stage, lymph node, vessel invasion and distant metastasis. Meanwhile, Cox's regression analysis revealed that uPA expression was an independent prognostic factor in patients with gastric cancer. In addition, previous studies demonstrated that uPA overexpression is associated with poor outcome in various types of cancer. For example, Horvatic et al (36) assessed 105 patients with differentiated thyroid carcinoma and normal matched tissues using ELISA, and identified that increased uPA levels represent an independent unfavorable prognostic factor in patients with differentiated thyroid carcinoma. Wu et al (37) reported that combination analysis of uPA and epithelial cadherin expression 
levels in laryngeal cancer may be useful for predicting tumor metastatic risk and patient prognosis. In the present study, patients with stage III and IV gastric cancer expressing increased uPA levels exhibited significantly poorer survival compared with uPA-negative patients, indicating that uPA may serve an important role in tumor progression and metastasis in gastric cancer.

A previous study demonstrated that RNA interference-mediated FOXM1 knockdown inhibits HeLa cell growth, with significantly decreased uPA levels in vitro (38). In addition, Ahmad et al (39) demonstrated a similar pattern in breast cancer cell lines. However, the association between FOXM1 levels and uPA expression levels in patients with gastric cancer remains incompletely understood. The results of the present study demonstrated that FOXM1 upregulation leads to uPA overexpression in gastric cancer. Furthermore, Kaplan-Meier estimator survival analysis revealed that patients with tumors exhibiting increased expression levels of both FOXM1 and uPA exhibited significantly poorer prognosis compared with those coexpressing these proteins at decreased levels. These results suggested that FOXM1 and UPA may interact to promote tumorigenesis and their combined expression may be used as an independent prognostic marker in gastric cancer.

In conclusion, the results of the present study suggested that increased FOXM1 and UPA expression levels in gastric cancer are significantly associated with aggressive progression and poor prognosis. Thus, overexpression of FOXM1 and uPA may possess potential diagnostic value and indicate poor prognosis in patients with gastric cancer.

\section{Acknowledgements}

The present study was supported by the National Natural Science Foundation of China (grant nos. 81502090 and 81470109), the Zhejiang Provincial Natural Science Foundation of China (grant no. LY14H160039) and the Medicine and Health Research Foundation of Zhejiang (grant no. 2013KYB022).

\section{References}

1. Ferlay J, Shin HR, Bray F, Forman D, Mathers C and Parkin DM: Estimates of worldwide burden of cancer in 2008: GLOBOCAN 2008. Int J Cancer 127: 2893-2917, 2010.

2. Yang L: Incidence and mortality of gastric cancer in China. World J Gastroenterol 12: 17-20, 2006.

3. Wang J, Dang P, Raut CP, Pandalai PK, Maduekwe UN, Rattner DW, Lauwers GY and Yoon SS: Comparison of a lymph node ratio-based staging system with the 7th AJCC system for gastric cancer: Analysis of 18,043 patients from the SEER database. Ann Surg 255: 478-485, 2012.

4. Nashimoto A: Current status of treatment strategy for elderly patients with gastric cancer. Int J Clin Oncol 18: 969-970, 2013.

5. Nadauld LD, Garcia S, Natsoulis G, Bell JM, Miotke L, Hopmans ES, Xu H, Pai RK, Palm C, Regan JF, et al Metastatic tumor evolution and organoid modeling implicate TGFBR2 as a cancer driver in diffuse gastric cancer. Genome Biol 15: 428, 2014.

6. Keighley MR: Gastrointestinal cancers in Europe. Aliment Pharmacol Ther 18 (Suppl 3): S7-S30, 2003.

7. Fang N, Zhang HQ, He B, Xie M, Lu S, Wan YY and Wang NR: Clinicopathological characteristics and prognosis of gastric cancer with malignant ascites. Tumour Biol 35: 3261-3268, 2014.
8. Koizumi W, Narahara H, Hara T, Takagane A, Akiya T, Takagi M, Miyashita K, Nishizaki T, Kobayashi O, Takiyama W, et al: S-1 plus cisplatin versus S-1 alone for first-line treatment of advanced gastric cancer (SPIRITS trial): A phase III trial. Lancet Oncol 9: 215-221, 2008.

9. Wierstra I and Alves J: FOXM1, a typical proliferation-associated transcription factor. Biol Chem 388: 1257-1274, 2007.

10. Wang IC, Chen YJ, Hughes D, Petrovic V, Major ML, Park HJ, Tan Y, Ackerson T and Costa RH: Forkhead box M1 regulates the transcriptional network of genes essential for mitotic progression and genes encoding the SCF (Skp2-Cks1) ubiquitin ligase. Mol Cell Biol 25: 10875-10894, 2005.

11. Costa RH: FoxM1 dances with mitosis. Nat Cell Biol 7: 108-110, 2005.

12. Kopanja D, Pandey A, Kiefer M, Wang Z, Chandan N, Carr JR, Franks R, Yu DY, Guzman G, Maker A and Raychaudhuri P: Essential roles of FoxM1 in Ras-induced liver cancer progression and in cancer cells with stem cell features. J Hepatol 63: 429-436, 2015

13. Zhang J, Zhang J, Cui X, Yang Y, Li M, Qu J, Li J and Wang J: FoxM1: A novel tumor biomarker of lung cancer. Int J Clin Exp Med 8: 3136-3140, 2015.

14. Bergamaschi A, Madak-Erdogan Z, Kim YJ, Choi YL, Lu H and Katzenellenbogen BS: The forkhead transcription factor FOXM1 promotes endocrine resistance and invasiveness in estrogen receptor-positive breast cancer by expansion of stem-like cancer cells. Breast Cancer Res 16: 436, 2014.

15. Wan X, Yeung C, Kim SY, Dolan JG, Ngo VN, Burkett S, Khan J, Staudt LM and Helman LJ: Identification of FoxM1/Bub1b signaling pathway as a required component for growth and survival of rhabdomyosarcoma. Cancer Res 72: 5889-5899, 2012.

16. Ferraris GM and Sidenius N: Urokinase plasminogen activator receptor: A functional integrator of extracellular proteolysis, cell adhesion, and signal transduction. Semin Thromb Hemost 39: 347-355, 2013.

17. Sidenius N and Blasi F: The urokinase plasminogen activator system in cancer: Recent advances and implication for prognosis and therapy. Cancer Metastasis Rev 22: 205-222, 2003.

18. Duffy MJ: The urokinase plasminogen activator system: Role in malignancy. Curr Pharm Des 10: 39-49, 2004.

19. Rabbani SA and Mazar AP: The role of the plasminogen activation system in angiogenesis and metastasis. Surg Oncol Clin N Am 10: 393-415, x, 2001.

20. Duffy MJ, Maguire TM, McDermott EW and O'Higgins N: Urokinase plasminogen activator: A prognostic marker in multiple types of cancer. J Surg Oncol 71: 130-135, 1999.

21. Choong PF and Nadesapillai AP: Urokinase plasminogen activator system: A multifunctional role in tumor progression and metastasis. Clin Orthop Relat Res (415 Suppl): S46-S58, 2003.

22. Plebani M, Herszènyi L, Carraro P, De Paoli M, Roveroni G, Cardin R, Tulassay Z, Naccarato R and Farinati F: Urokinase-type plasminogen activator receptor in gastric cancer: Tissue expression and prognostic role. Clin Exp Metastasis 15: 418-425, 1997.

23. Hu B, El Hajj N, Sittler S, Lammert N, Barnes R and Meloni-Ehrig A: Gastric cancer: Classification, histology and application of molecular pathology. J Gastrointest Oncol 3: 251-261, 2012

24. Halasi M and Gartel AL: FOX(M1) news-it is cancer. Mol Cancer Ther 12: 245-254, 2013.

25. Zeng J, Wang L, Li Q, Li W, Björkholm M, Jia J and Xu D: FoxM1 is up-regulated in gastric cancer and its inhibition leads to cellular senescence, partially dependent on p27 kip1. J Pathol 218: 419-427, 2009.

26. Okada K, Fujiwara Y, Takahashi T, Nakamura Y, Takiguchi S, Nakajima K, Miyata H, Yamasaki M, Kurokawa Y, Mori M and Doki Y: Overexpression of forkhead box M1 transcription factor (FOXM1) is a potential prognostic marker and enhances chemoresistance for docetaxel in gastric cancer. Ann Surg Oncol 20: 1035-1043, 2013.

27. Li X, Qi W, Yao R, Tang D and Liang J: Overexpressed transcription factor FOXM1 is a potential diagnostic and adverse prognostic factor in postoperational gastric cancer patients. Clin Transl Oncol 16: 307-314, 2014.

28. Hui MK, Chan KW, Luk JM, Lee NP, Chung Y, Cheung LC, Srivastava G, Tsao SW, Tang JC and Law S: Cytoplasmic Forkhead box M1 (FoxM1) in esophageal squamous cell carcinoma significantly correlates with pathological disease stage. World J Surg 36: 90-97, 2012. 
29. Chen $\mathrm{CH}$, Chien $\mathrm{CY}$, Huang CC, Hwang CF, Chuang HC, Fang FM, Huang HY, Chen CM, Liu HL and Huang CY: Expression of FLJ10540 is correlated with aggressiveness of oral cavity squamous cell carcinoma by stimulating cell migration and invasion through increased FOXM1 and MMP-2 activity. Oncogene 28: 2723-2737, 2009.

30. Wang Z, Banerjee S, Kong D, Li Y and Sarkar FH: Down-regulation of Forkhead Box M1 transcription factor leads to the inhibition of invasion and angiogenesis of pancreatic cancer cells. Cancer Res 67: 8293-8300, 2007.

31. Li Q, Zhang N, Jia Z, Le X, Dai B, Wei D, Huang S, Tan D and Xie K: Critical role and regulation of transcription factor FoxM1 in human gastric cancer angiogenesis and progression. Cancer Res 69: 3501-3509, 2009.

32. Petersen LC, Lund LR, Nielsen LS, Danø K and Skriver L: One-chain urokinase-type plasminogen activator from human sarcoma cells is a proenzyme with little or no intrinsic activity. J Biol Chem 263: 11189-11195, 1988.

33. Bekes EM, Deryugina EI, Kupriyanova TA, Zajac E, Botkjaer KA, Andreasen PA and Quigley JP: Activation of pro-uPA is critical for initial escape from the primary tumor and hematogenous dissemination of human carcinoma cells. Neoplasia 13: 806-821, 2011.

34. Tang L and Han X: The urokinase plasminogen activator system in breast cancer invasion and metastasis. Biomed Pharmacother 67: 179-182, 2013.
35. Heiss MM, Simon EH, Beyer BC, Gruetzner KU, Tarabichi A, Babic R, Schildberg FW and Allgayer H: Minimal residual disease in gastric cancer: Evidence of an independent prognostic relevance of urokinase receptor expression by disseminated tumor cells in the bone marrow. J Clin Oncol 20: 2005-2016, 2002.

36. Horvatic Herceg G, Herceg D, Kralik M, Kulic A, Bence-Zigman Z, Tomic-Brzac H, Bracic I, Kusacic-Kuna S and Prgomet D: Urokinase plasminogen activator and its inhibitor type-1 as prognostic factors in differentiated thyroid carcinoma patients. Otolaryngol Head Neck Surg 149: 533-540, 2013.

37. Wu HY, Shen XH, Ni RS, Qian XY and Gao X: Expression of E-cadherin and uPA and their prognostic value in carcinoma of human larynx. Zhonghua Er Bi Yan Hou Tou Jing Wai Ke Za Zhi 44: 1024-1028, 2009 (In Chinese).

38. Chen H, Zou Y, Yang H, Wang J and Pan H: Downregulation of FoxM1 inhibits proliferation, invasion and angiogenesis of $\mathrm{HeLa}$ cells in vitro and in vivo. Int J Oncol 45: 2355-2364, 2014.

39. Ahmad A, Wang Z, Kong D, Ali S, Li Y, Banerjee S, Ali R and Sarkar FH: FoxM1 down-regulation leads to inhibition of proliferation, migration and invasion of breast cancer cells through the modulation of extra-cellular matrix degrading factors. Breast Cancer Res Treat 122: 337-346, 2010. 